\begin{tabular}{|c|l|}
\hline Title & Nonlinear dynamical effects on reaction rates in thermally fluctuating environments \\
\hline Author(s) & Kawai, Shinnosuke; Komatsuzaki, Tamiki \\
\hline Citation & $\begin{array}{l}\text { Physical Chemistry Chemical Physics, 12(27), 7636-7647 } \\
\text { https://doi.org/10.1039/0922596j }\end{array}$ \\
\hline Issue Date & $2010-07-21$ \\
\hline Doc URL & http://hdl.handle.net/2115/45146 \\
\hline Rights & Phys. Chem. Chem. Phys., 2010, 12, 7636-7647- Reproduced by permission of the PCCP Owner Societies \\
\hline Type & article (author version) \\
\hline File Information & PCCP12-27_7636-7647.pdf \\
\hline
\end{tabular}

Instructions for use 


\title{
Nonlinear Dynamical Effects on Reaction Rates in Thermally Fluctuating Environments
}

\author{
Shinnosuke Kawai ${ }^{1 *}$ and Tamiki Komatsuzaki ${ }^{1,2}$ \\ ${ }^{1}$ Molecule \& Life Nonlinear Sciences Laboratory, \\ Research Institute for Electronic Science, \\ Hokkaido University, Kita 20 Nishi 10, \\ Kita-ku, Sapporo 001-0020, Japan \\ and \\ ${ }^{2}$ Core Research for Evolutional Science and Technology (CREST), \\ Japan Science and Technology Agency (JST), \\ Kawaguchi, Saitama 332-0012, Japan \\ * Research Fellow of the Japan Society for the Promotion of Science
}

An analytical framework to scrutinize the physical origin, especially nonlinear dynamical effects, of rate constants in thermal fluctuation is developed.

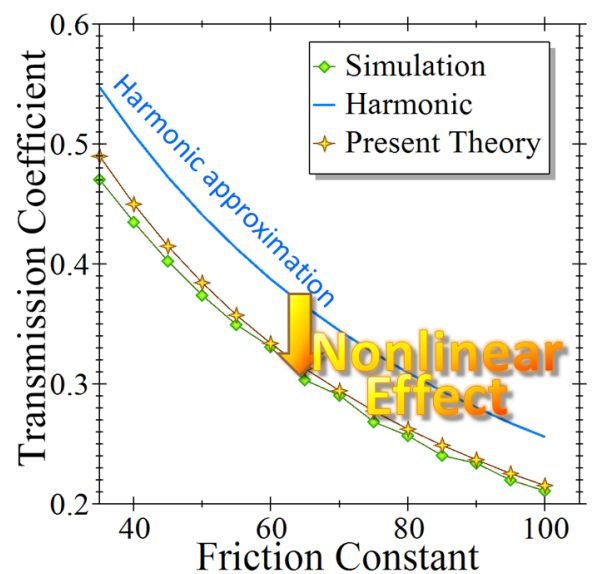

A framework to calculate the rate constants of condensed phase chemical reactions of manybody systems is presented without relying on the concept of transition state. The theory is based on a framework we developed recently adopting multidimensional underdamped Langevin equation in the region of rank-one saddle. The theory provides a reaction coordinate expressed as an analytical nonlinear functional of the position coordinates and velocities of the system (solute), the friction constants, and the random force of the environment (solvent). Up to moderately high temperature, the sign of the reaction coordinate can determine the final destination of the reaction in a thermally fluctuating media, irrespective of what values the other (nonreactive) coordinates may take. In this paper, it is shown that the reaction probability is analytically derived as the probability of the reaction coordinate being positive, and that the integration with the Boltzmann distribution of the initial conditions leads to the exact reaction rate constant when the local equilibrium holds and the quantum effect is negligible. Because of analytical nature of the theory taking into account all nonlinear effects and their combination with fluctuation and dissipation, the theory naturally provides us with the firm mathematical foundation of the origin of the reactivity of the reaction in a fluctuating media.

\section{INTRODUCTION}

Chemical reaction is one of the most important subjects of natural science through a wide range of fields not only just for chemistry but also for celestial mechanics, ${ }^{1-3}$ atomic physics, ${ }^{4,5}$ cluster physics, ${ }^{6}$ environmental science, ${ }^{7}$ and biology. ${ }^{8}$ In the studies of chemical reactions, one of the central concepts is the rate constant, the proportion of the number of the species (molecules) in the reactant state reacting to form the products per unit time. The reactant and the product (states) correspond to distinct regions of the phase space (that is, the space spanned by the position coordinates and the velocities) of the system, with the rate given by the flux from the former region to the latter. In principle, the rate can differ from portion by portion in the reactant region of phase space. ${ }^{9}$ The existence of the reaction rate constant relies on the assumption of local equilibrium, that is, the system fully explores a region of the phase space assigned as the reactant before it crosses from the region to that of the product in the phase space. This also results in the time scale separation between that of the exploration through the reactant and that of crossing from the reactant to the product: the time scale is much longer in the former than that in the latter. Whenever one calculates the rate constant of chemical reactions, the local equilibrium needs to hold in the region of the phase space assigned as the reactant. ${ }^{10,11}$ To calculate the rate constant chemists have long developed a concept of 'transition state (TS). ${ }^{12-19}$ It was originally defined as a dividing hypersurface through which all the reacting trajectories must cross once and only once before being "captured" in the product. 
The non-recrossing property, if the assumption holds, enables to identify the reaction rate with the flux through the no-return TS without tracing the future of each trajectory (i.e., no need to check how many times the system crosses the surface assigned as the TS). Moreover, if the reaction coordinate, lying transverse to the TS, is decoupled from the other coordinates of the system, its value can tell us the final destination of the given initial condition (i.e., whether the system will proceed to the product or go back to the reactant) without referring the other nonreactive coordinates.

However, since manybody reacting systems generically possess nonlinear couplings among the modes, the very existence of no-return TS and the reaction coordinate decoupled from all the rest is not a trivial issue. It was just recently that the existence of the (no-return) TS was established with a firm mathematical ground ${ }^{4-6,20-35}$ (one can also see several reviews ${ }^{10,36-42}$ and books ${ }^{43,44}$ ). The crux is the construction of nonlinear coordinate transformation with which one of the new coordinates is unstable and decoupled from the other coordinates at some moderate conditions. The no-return TS is then given by an equation setting the new reaction coordinate to be zero, which also enables us to tell the final destination of the reaction system without performing trajectory calculation. Their method to take into account anharmonicity and nonlinear couplings among modes in the passage through the region of rank-one saddle is based on a perturbation theory, the so-called normal form (NF) theory. ${ }^{45}$ The validity of the usage of perturbation theory to take into account such nonlinearity in the region of rank-one saddle has been ensured by several studies in experiments ${ }^{46,47}$ and theories ${ }^{48-57}$ on the regularity of crossing dynamics over the saddle and the corresponding phase space geometrical structure (e.g., a no-return TS) in a wide class of Hamiltonian systems. ${ }^{4-6,20-35}$ One can naturally adopt this perturbation theory without loss of generality as far as the total energy of the system is not so very high that any perturbation treatment is invalidated. These developments, however, are all based on the Hamiltonian formalism, which corresponds to isolated systems (i.e., gas phase).

Most of reactions in organic synthesis and chemical biology occur in condensed phase under the existence of external forces from the surroundings of the system. The external forces exerted by the environment (e.g., solvent) are often regarded as being "stochastic" so that the molecule (unpredictably) experiences many recrossings over the saddle due to the fluctuating force. Kramers ${ }^{58}$ described the reaction dynamics as a stochastic process in the framework of underdamped Langevin equation along a chosen, onedimensional reaction coordinate. Followed by Kramers, Grote and Hynes ${ }^{59}$ formulated condensed phase reaction in terms of underdamped generalized Langevin equation. In their frameworks, the effect of nonlinear couplings among multiple degrees of freedom of the system (e.g., solute) is not explicitly taken into account [all the nonlinear effects are put into the potential of mean force, random forces, and memory (kernel)]. Sumi and Marcus ${ }^{60,61}$ revealed that the inclusion of the other degrees of freedom in addition to a one-dimensional reaction coordinate is essential for understanding the viscosity dependency observed in many experiments ranging from electro- chemical to enzymatic reactions. However, there still remains the fundamental question of along what reaction coordinate a complex system with nonlinearly coupled multiple modes actually follows under the disturbance of thermal fluctuation.

Very recently, we have incorporated the non-Hamiltonian NF theory ${ }^{62}$ together with the time-dependent formulation given by Ref. 63 to multidimensional underdamped Langevin equation without assuming the form and the dimension of potential of mean force. ${ }^{64-66}$ The new reaction coordinate is obtained with its analytical expression as a nonlinear functional of the position coordinates and velocities of the system (solute), the friction constants, and the random force of the environment (solvent). Up to moderately high temperature, the reaction coordinate is found to be decoupled from the other nonreactive coordinates and the no-return TS can be defined in principle free from the recrossing problem even under the existence of thermal noise. At temperature increases further, the no-return TS starts to cease. Nevertheless, the sign of the new reaction coordinate can solely determine the final destination of the reaction without referring the other nonreactive coordinates.

In this paper, we present an analytical formulation of condensed phase reaction rate by the integration of the reaction probability naturally obtained from the reaction coordinate with the Boltzmann distribution of the initial conditions in the saddle region. This formulation explicitly takes into account the nonlinearity and the multiple degrees of freedom and leads to the exact reaction rate as far as the local equilibrium holds and the quantum effect is negligible. As an illustrative example, we analyze the Müller-Brown potential of multiple saddles for a range of friction.

\section{THEORY}

\section{A. Normal form theory}

First we present a brief summary of the theory developed in previous papers ${ }^{64-66}$ of the dynamical structure buried in a thermally fluctuating environment. As the description of the reaction, we adopt a multi-dimensional underdamped Langevin equation:

$$
\ddot{q}_{j}=-\frac{\partial U}{\partial q_{j}}-\sum_{i=1}^{n} \gamma_{i j} \dot{q}_{i}+\xi_{j}(t),
$$

where $\left(q_{1}, q_{2}, \ldots, q_{n}\right)$ are position coordinates of the system, with $n$ being the number of degrees of freedom of the system, $U$ the potential of mean force, $\gamma_{i j}$ 's friction coefficients and $\xi_{j}(t)$ 's describe the fluctuating force from the environment such as solvent. Given the reaction system, we assume the existence of a rank-one saddle to link the reactants and the products regions on the potential of mean force. Without loss of generality, the origin $(q=0)$ of the coordinate system is set to be at the saddle point. The fluctuating force $\xi_{j}(t)$ has a Gaussian distribution with zero mean, with their variance given by the fluctuation-dissipation theorem

$$
\left\langle\xi_{i}(t) \xi_{j}\left(t^{\prime}\right)\right\rangle=2 k_{\mathrm{B}} T \gamma_{i j} \delta\left(t-t^{\prime}\right),
$$


where $T$ is the temperature, $k_{\mathrm{B}}$ Boltzmann constant, and $\delta$ Dirac's delta function, respectively. For simplicity, we treat the case $\gamma_{1 j}=0$ (for $j=2, \ldots, n$ ), that is, the cross terms in the matrix $\left(\gamma_{i j}\right)$ are zero between the reactive mode (mode 1 ) and the non-reactive modes. Extension to general cases is more involved but straightforward.

We separate the potential $U$ into harmonic and anharmonic terms:

$$
\begin{gathered}
U(q)=-\frac{\omega^{\ddagger^{2}}}{2} q_{1}^{2}+\sum_{j=2}^{n} \frac{\omega_{j}^{2}}{2} q_{j}^{2}+U_{\mathrm{NL}}(q), \\
U_{\mathrm{NL}}(q)=\sum_{k=1}^{\infty} \varepsilon^{k} \sum_{|m|=2+k} \alpha_{m} q_{1}^{m_{1}} \cdots q_{n}^{m_{n}},
\end{gathered}
$$

where $\omega_{j}$ 's are harmonic frequencies of the non-reactive vibrational modes, $\omega^{\ddagger^{2}}$ is the curvature along the reactive mode at the origin (the saddle point). The anharmonic part is expressed as polynomials of cubic and higher order with expansion coefficients $\alpha_{m}$. In Eq. (3), the formal parameter $\varepsilon$ marks the order of perturbation. The $k$-th order of perturbation is given by the terms with $|m|=m_{1}+\cdots+m_{n}=2+k$ because the zeroth order corresponds to the harmonic approximation $(|m|=2)$.

In the framework we developed recently, ${ }^{64-66}$ we introduced a nonlinear coordinate transformation $\left(q_{1}, \ldots, q_{n}, \dot{q}_{1}, \ldots, \dot{q}_{n}\right) \longmapsto\left(y_{1}, \ldots, y_{2 n}\right) \quad$ to transform the equations of motion, Eq. (1), into a form as simpler as possible. As the final form of the equation of motion, we have proposed two types. ${ }^{66}$ One is called "partial NF," having the following equations of motion:

$$
\begin{aligned}
& \dot{y}_{1} \approx\left\{\lambda_{1}+c_{1}(t)\right\} y_{1}, \\
& \dot{y}_{j} \approx \lambda_{j} y_{j}+c_{j}\left(y_{1}, \ldots, y_{2 n}, t\right), \quad j=2,3, \ldots, 2 n
\end{aligned}
$$

where $\lambda_{1}$ is a positive number determined by $\omega^{\ddagger}$ and $\gamma_{11}$. Here the coefficient $c_{1}(t)$ depends only on time $t$. The functions $c_{j}\left(y_{1}, \ldots, y_{2 n}, t\right)(j=2,3, \ldots, 2 n)$ are allowed to take any functional form. In the partial NF, the evolution of the coordinate $y_{1}$ is independent of the other coordinate. The other type of NF is called "minimal NF"

$$
\begin{aligned}
& \dot{y}_{1} \approx\left\{\lambda_{1}+c_{1}(y, t)\right\} y_{1}, \\
& \dot{y}_{j} \approx \lambda_{j} y_{j}+c_{j}\left(y_{1}, \ldots, y_{2 n}, t\right), \quad j=2,3, \ldots, 2 n
\end{aligned}
$$

Here we allow the dependence of $c_{1}(y, t)$ on the other coordinates. The minimal NF is found to have better convergence property than the partial NF. ${ }^{66}$

Since the transformation is constructed by a perturbation theory, ${ }^{64-66}$ the coefficient $c_{1}(y, t)$ is also expanded in perturbation series

$$
c_{1}(y, t)=\sum_{k=1}^{N} \varepsilon^{k} c_{1}^{(k)}(y, t)+O\left(\varepsilon^{N+1}\right) .
$$

where $N$ is the order we perform the perturbation calculation. Note the expansion for $c_{1}(y, t)$ begins with order one in $\varepsilon$. Since $\lambda_{1}$ is of order zero, it is usually expected that $c_{1}(y, t)$ does not exceed $\lambda_{1}$ in magnitude, that is, the motion along $y_{1}$-direction is unstable. The unstable direction corresponds to the motion sliding down the barrier when time $t \rightarrow+\infty$. In both the cases of partial and minimal NF, $y_{1}=0$ yields an invariant set (i.e., $d y_{1} / d t=0$ if $y_{1}=0$ ). That is, once the system is at the manifold of $y_{1}=0$, the system will remain to stay there for $t \rightarrow \pm \infty$. In the other words, if $y_{1}\left(t_{0}\right)>0$ or $y_{1}\left(t_{0}\right)<0$ at any time $t_{0}$, the sign of $y_{1}$ will never change because the system should cross the manifold of $y_{1}=0$ in order to change the sign of $y_{1}$ (it is prohibited as far as the form of Eq. (5) holds). The sign of $y_{1}$ remains the same for all the time, with the absolute value increasing infinitely. Thus, the sign of $y_{1}$ at any instantaneous time in the region of saddle determines whether the system escapes from the vicinity of the saddle to the reactant or the product directions.

The transformed coordinate $y_{1}$ can be written in the form of polynomials in the original coordinates $(q, \dot{q})$ :

$$
\begin{aligned}
y_{1}= & a_{1} q_{1}+a_{2} \dot{q}_{1}-S\left[\lambda_{1}, \tilde{\xi}_{1}\right](t)+F_{0}[\xi](t) \\
& +\sum_{|m| \geq 2} w_{m} q_{1}{ }^{m_{1}} \cdots q_{n}{ }^{m_{n}} \dot{q}_{1}^{m_{n+1}} \cdots \dot{q}_{n}^{m_{2 n}} \\
& +\sum_{|m| \geq 1} F_{m}[\xi](t) q_{1}{ }^{m_{1}} \cdots q_{n}{ }^{m_{n}} \dot{q}_{1}^{m_{n+1}} \cdots \dot{q}_{n}^{m_{2 n}}
\end{aligned}
$$

The zeroth order part of $y_{1}$ is given as a linear combination of the position $q_{1}$ and the velocity $\dot{q}_{1}$ along the reactive direction, and a time-dependent shift that is a linear functional of the random force $\tilde{\xi}_{1}(t)$. The coefficients $a_{1}, a_{2}$, and $\lambda$ are given by the eigenvector and the eigenvalues of the linear part of Eq. (1), and therefore depend on the friction constants. The symbol $S$ is defined by ${ }^{63,64}$

$$
S[\mu, \psi](t) \stackrel{\text { def }}{=}\left\{\begin{array}{cc}
\int_{-\infty}^{0} \exp (-\mu \tau) \psi(t+\tau) d \tau & (\operatorname{Re} \mu<0) \\
-\int_{0}^{+\infty} \exp (-\mu \tau) \psi(t+\tau) d \tau & (\operatorname{Re} \mu>0)
\end{array}\right.
$$

for any complex number $\mu$ and function $\psi(t)$. The higher order terms with $w_{m}$ come from the nonlinearity in the system. The time-dependent coefficients $F_{0}[\xi](t)$ and $F_{m}[\xi](t)$ are nonlinear functionals of the random force $\xi(t)$. Their functional forms are determined by the properties of the system including the friction constants $\gamma$ and the nonlinear parts of the potential. They thus can be regarded as combined effects of the nonlinearity of the system and the random force from the environment. Each coefficient is expanded as a power series in the perturbation parameter $\varepsilon$ :

$$
\begin{gathered}
w_{m}=\sum_{k=1}^{N} \varepsilon^{k} w_{m}^{(k)}+O\left(\varepsilon^{N+1}\right), \\
F_{0}[\xi](t)=\sum_{k=1}^{N} \varepsilon^{k} F_{0}^{(k)}[\xi](t)+O\left(\varepsilon^{N+1}\right), \\
F_{m}[\xi](t)=\sum_{k=1}^{N} \varepsilon^{k} F_{m}^{(k)}[\xi](t)+O\left(\varepsilon^{N+1}\right) .
\end{gathered}
$$

All these three groups of the coefficients in the nonlinear part depend on the friction constants $\gamma$ although not written explicitly. ${ }^{64}$ 
The zeroth order part is given analytically in the case of $\gamma_{1 j}=0$ (for $j=2, \ldots, n$ ): we normalize the following linear equation

$$
\frac{d}{d t}\left(\begin{array}{c}
q_{1} \\
\dot{q}_{1}
\end{array}\right)=\left(\begin{array}{cc}
0 & 1 \\
\omega^{\ddagger^{2}} & -\gamma_{11}
\end{array}\right)\left(\begin{array}{l}
q_{1} \\
\dot{q}_{1}
\end{array}\right)+\left(\begin{array}{c}
0 \\
\xi_{1}(t)
\end{array}\right) .
$$

The positive eigenvalue is given by

$$
\lambda_{1}=\left(-\gamma_{11}+\sqrt{4 \omega^{\ddagger^{2}}+\gamma_{11}^{2}}\right) / 2,
$$

and the corresponding linear coefficients can be taken as

$$
\begin{aligned}
& a_{1}=\left(\gamma_{11}+\sqrt{4 \omega^{\ddagger^{2}}+\gamma_{11}^{2}}\right) / 2, \\
& a_{2}=1 .
\end{aligned}
$$

This implies that as the friction constant increases the eigenvalue $\lambda_{1}$ of the reactive direction becomes smaller and the contribution of the position coordinate $q_{1}$ to the reaction coordinate $y_{1}$ (at the lowest order) becomes more significant than that of the velocity $\dot{q}_{1}$.

\section{B. Reaction probability}

Here we derive an analytical expression of the reaction probability using the new reaction coordinate $y_{1}$ and the statistical properties of the random force $\xi(t)$. For a given initial condition $(q, \dot{q})$, the value of $y_{1}$ has an average value $\left\langle y_{1}\right\rangle$ and fluctuation around it, reflecting the stochastic nature of the random force $\xi(t)$. The probability for the reaction is given by the probability of $y_{1}>0$. Let us denote the probability distribution of $y_{1}$ by $P\left(y_{1}\right)$, i.e., the probability that the value of $y_{1}$ falls into the range $a<y_{1}<b$ is given by the integration

$$
\int_{a}^{b} P\left(y_{1}\right) d y_{1} \text {. }
$$

The distribution can be characterized by its mean value

$$
m \stackrel{\text { def }}{=}\left\langle y_{1}\right\rangle=\int_{-\infty}^{+\infty} y_{1} P\left(y_{1}\right) d y_{1}
$$

and the moments around it $^{67}$

$$
\mu_{n} \stackrel{\text { def }}{=}\left\langle\left(y_{1}-m\right)^{n}\right\rangle=\int_{-\infty}^{+\infty}\left(y_{1}-m\right)^{n} P\left(y_{1}\right) d y_{1} .
$$

The second moment $\mu_{2}$ is related to the standard deviation $\sigma$ by

$$
\sigma^{2}=\left\langle\left(y_{1}-m\right)^{2}\right\rangle=\mu_{2} .
$$

The third moment divided by $\sigma^{3}$ is known as skewness

$$
\gamma^{\prime}=\frac{\mu_{3}}{\sigma^{3}}
$$

The analytical expressions for $y_{1}{ }^{n}$ contains combinations of stochastic forces $\xi_{j}(t)$ wrapped by $S$-functionals [Eq. (8)].
With the statistical properties of $\xi_{j}$ given by Eq. (2), their average can be calculated by the following formulas ${ }^{64}$ :

$$
\begin{aligned}
\left\langle S\left[\lambda_{i}, \xi_{j}\right]\right\rangle & =0, \\
\left\langle S\left[\lambda_{1}, \xi_{i}\right] S\left[\lambda_{2}, \xi_{j}\right]\right\rangle & =2 k_{\mathrm{B}} T \gamma_{i j} F\left(\lambda_{1}, \lambda_{2}\right), \\
\left\langle S\left[\lambda_{3}, S\left[\lambda_{1}, \xi_{i}\right] S\left[\lambda_{2}, \xi_{j}\right]\right]\right\rangle & =-2 k_{\mathrm{B}} T \lambda_{3}^{-1} \gamma_{i j} F\left(\lambda_{1}, \lambda_{2}\right),
\end{aligned}
$$

with the symbol $F$ defined by

$$
F\left(\lambda_{1}, \lambda_{2}\right) \stackrel{\text { def }}{=}\left\{\begin{array}{cl}
\frac{1}{\lambda_{1}+\lambda_{2}} & \left(\text { if } \operatorname{Re} \lambda_{1}>0 \text { and } \operatorname{Re} \lambda_{2}>0\right) \\
-\frac{1}{\lambda_{1}+\lambda_{2}} & \left(\text { if } \operatorname{Re} \lambda_{1}<0 \text { and } \operatorname{Re} \lambda_{2}<0\right) \\
0 & \text { (otherwise) }
\end{array}\right.
$$

Some formulations arising in higher orders are also available. $^{64}$

From the information of the moments, the probability distribution can be approximated by Gram-Charlier A series, ${ }^{67}$ which is a series expansion consisting of Gaussian distribution plus correction terms due to higher order moments. Note here that in the harmonic approximation $(\varepsilon=0)$ the distribution is exactly Gaussian if the random force $\xi$ has Gaussian distribution. First we define a scaled variable $\tilde{y}_{1}$ by

$$
\tilde{y}_{1} \stackrel{\text { def }}{=} \frac{y_{1}-m}{\sigma} \text {. }
$$

Then the probability distribution for $\tilde{y}_{1}$ can be expanded as

$$
\tilde{P}\left(\tilde{y}_{1}\right)=\left[1+\sum_{n=3}^{\infty} \frac{c_{n}}{n !} H_{n}\left(\tilde{y}_{1}\right)\right] \frac{\exp \left(-\tilde{y}_{1}^{2} / 2\right)}{\sqrt{2 \pi}},
$$

where $H_{n}$ is the probabilists' Hermite polynomials defined by

$$
H_{n}(z)=(-1)^{n} \exp \left(z^{2} / 2\right)\left(\frac{d}{d z}\right)^{n} \exp \left(-z^{2} / 2\right)
$$

$(z \in \mathbb{R})$ and the expansion coefficients $c_{n}$ are given by

$$
c_{n}=\left\langle H_{n}\left(\tilde{y}_{1}\right)\right\rangle .
$$

For example, the cubic part is given by

$$
c_{3}=\left\langle\tilde{y}_{1}^{3}-3 \tilde{y}_{1}\right\rangle=\sigma^{-3}\left(\left\langle y_{1}{ }^{3}\right\rangle-3 \sigma^{2} m-m^{3}\right)=\gamma^{\prime},
$$

where $\gamma^{\prime}$ is the skewness defined in Eq. (17). Since the reaction probability is the probability for $y_{1}>0$, which is equivalent with $\tilde{y}_{1}>-m / \sigma$, it is given by

$$
\begin{aligned}
P_{\text {reaction }}= & \int_{-m / \sigma}^{+\infty} \tilde{P}\left(\tilde{y}_{1}\right) d \tilde{y}_{1} \\
= & \frac{1}{2}\left[1+\operatorname{Erf}\left(\frac{m}{\sqrt{2} \sigma}\right)\right] \\
& +\sum_{n=3}^{\infty} \frac{c_{n}}{n !} \frac{1}{\sqrt{2 \pi}} H_{n-1}\left(-\frac{m}{\sigma}\right) \exp \left(-\frac{m^{2}}{2 \sigma^{2}}\right),
\end{aligned}
$$

where the error function is defined by

$$
\operatorname{Erf}(z)=\frac{2}{\sqrt{\pi}} \int_{0}^{z} \exp \left(-z^{2}\right) d z
$$




\section{Transmission coefficient}

The transmission coefficient $\kappa$ is defined as the ratio of the true rate constant to that by transition state theory (TST). Except the quantum effect and under the assumption of local equilibrium, it is given by ${ }^{68,69}$

$$
\kappa=\lim _{t \rightarrow+\infty} \frac{\left\langle\dot{q}_{1}(0) \theta\left(q_{1}(t)\right)\right\rangle}{\left\langle\dot{q}_{1}(0) \theta\left(\dot{q}_{1}(0)\right)\right\rangle},
$$

where $q_{1}$ is the naïve reaction coordinate, often obtained as the unstable normal mode at the top of the saddle, $\theta$ is a step function, and the angular bracket denotes the thermal average at the surface $q_{1}=0$ :

$$
\langle A(q, \dot{q})\rangle \stackrel{\text { def }}{=} \int A(q, \dot{q}) \delta\left(q_{1}\right) \exp \left(-E(q, \dot{q}) / k_{\mathrm{B}} T\right) d q d \dot{q},
$$

for any function $A$ of $(q, \dot{q})$, with $E$ being the total energy of the system.

The step function $\theta\left(q_{1}(t)\right)$ in the numerator in Eq. (27) extracts those trajectories which arrived in the product region for sufficiently large time $t$, which requires many trajectory calculations for all initial conditions in the ensemble for dissipative reaction systems under stochastic, thermal fluctuation. The step function $\theta\left(\dot{q}_{1}(0)\right)$ in the denominator corresponds to the assumption of TST that any trajectory crossing the TS in the positive direction is reactive. Here we present analytical expressions of these quantities and hence the transmission coefficient $\kappa$ by using the reaction probability Eq. (25) derived in Sec. II B. The ensemble average of $\theta\left(q_{1}(t)\right)$ can be calculated as the reaction probability by Eq. (25):

$$
\left\langle\dot{q}_{1}(0) \theta\left(q_{1}(t)\right)\right\rangle=\left\langle\dot{q}_{1} P_{\text {reaction }}(q, \dot{q})\right\rangle .
$$

We assume that the total energy is given as a sum of kinetic energy and potential of mean force:

$$
E(q, \dot{q})=\frac{1}{2} \sum_{j=1}^{n} \dot{q}_{j}^{2}-\frac{\omega^{\ddagger^{2}}}{2} q_{1}^{2}+\sum_{j=2}^{n} \frac{\omega_{j}^{2}}{2} q_{j}^{2}+U_{\mathrm{NL}}(q),
$$

where $U_{\mathrm{NL}}(q)$ is the nonlinear part of the potential given as polynomial expansion of cubic and higher order terms. By substituting Eqs. (25), (28), and (30), and by integration by parts, we obtain

$$
\begin{aligned}
& \left\langle\dot{q}_{1} P_{\text {reaction }}(q, \dot{q})\right\rangle \\
= & \frac{1}{(2 \pi)^{1 / 2}} \int\left[k_{\mathrm{B}} T \frac{\partial}{\partial \dot{q}_{1}}\left(\frac{m}{\sigma}\right)+\sum_{n=3}^{\infty} \frac{\dot{q}_{1}}{n !} c_{n} H_{n-1}\left(-\frac{m}{\sigma}\right)\right] \\
& \exp \left[-\frac{1}{k_{\mathrm{B}} T}\left\{\frac{1}{2} \dot{q}_{1}^{2}+\frac{1}{2} \sum_{j=2}^{n}\left(\dot{q}_{j}^{2}+\omega_{j}^{2} q_{j}^{2}\right)+U_{\mathrm{NL}}\right\}-\frac{m^{2}}{2 \sigma^{2}}\right] \\
& d \dot{q}_{1} d q_{\mathrm{b}} d \dot{q}_{\mathrm{b}},
\end{aligned}
$$

where

$$
q_{\mathrm{b}}=\left(q_{2}, q_{3}, \ldots, q_{n}\right)
$$

is the collection of the non-reactive modes.
Analytical expressions for $m$ and $\sigma^{2}$ are obtained from Eq. (7) by using Eqs. (2), (8), (11), (12), and also $\left\langle S\left[\lambda_{1}, \xi_{1}\right](t)^{2}\right\rangle=k_{\mathrm{B}} T \gamma_{11} / \lambda_{1}{ }^{64}$ : for $q_{1}=0$,

$$
\begin{aligned}
m= & \left\langle y_{1}\right\rangle \\
= & \dot{q}_{1} \\
& +\sum_{k=1}^{N} \varepsilon^{k} \sum_{|m| \leq 1+k} \mu_{m}^{(k)}(\gamma, T) q_{2}{ }^{m_{2}} \cdots q_{n}^{m_{n}} \dot{q}_{1}^{m_{n+1}} \cdots \dot{q}_{n}^{m_{2 n}} \\
& +O\left(\varepsilon^{N+1}\right)
\end{aligned}
$$

$$
\begin{aligned}
\sigma^{2}= & \left\langle\left(y_{1}-m\right)^{2}\right\rangle \\
= & \frac{k_{\mathrm{B}} T \gamma_{11}}{\lambda_{1}} \\
& +\sum_{k=1}^{N} \varepsilon^{k} \sum_{|m| \leq 2+k} s_{m}^{(k)}(\gamma, T) q_{2}{ }^{m_{2}} \cdots q_{n}{ }^{m_{n}} \dot{q}_{1}^{m_{n+1}} \cdots \dot{q}_{n}^{m_{2 n}} \\
& +O\left(\varepsilon^{N+1}\right),
\end{aligned}
$$

where $\mu_{m}^{(k)}(\gamma, T)$ and $s_{m}^{(k)}(\gamma, T)$ are thermal averages at temperature $T$ of the coefficients appearing in the expressions of $y_{1}$ [Eq. (7)] and $\left(y_{1}-m\right)^{2}$, respectively. Here we have written $\gamma$ to emphasize the dependence of these terms on the friction constants. These dependence originates from the $\gamma$ dependence of the coefficients appearing in the normal form transformation of Eq. (7).

Terms like $m / \sigma$ appearing in Eq. (31) can also be expressed as polynomials by using the expansion

$$
\frac{1}{\left(z_{0}+\delta z\right)^{1 / 2}}=\frac{1}{z_{0}^{1 / 2}}-\frac{1}{2 z_{0}^{3 / 2}}(\delta z)+\frac{3}{8 z_{0}^{5 / 2}}(\delta z)^{2}-\cdots
$$

with $\left(z_{0}+\delta z\right)$ substituted by $\sigma^{2}$. It is possible to identify $z_{0}$ with the zeroth order part of $\sigma^{2}$. However, we have found that the approximation gets better if we recollect the constant terms in Eq. (34):

$$
\begin{aligned}
& z_{0}=\frac{k_{\mathrm{B}} T \gamma_{11}}{\lambda_{1}}+\sum_{k=1}^{N} \varepsilon^{k} s_{0}^{(k)}(\gamma, T) \\
& \delta z=\sum_{k=1}^{N} \varepsilon^{k} \sum_{|m| \leq 2+k, m \neq 0} s_{m}^{(k)}(\gamma, T) q_{2}{ }^{m_{2}} \cdots q_{n}{ }^{m_{n}} \dot{q}_{1}^{m_{n+1}} \cdots \dot{q}_{n}^{m_{2 n}}
\end{aligned}
$$


We now rewrite Eq. (31) as

$$
\begin{aligned}
& \left\langle\dot{q}_{1} P_{\text {reaction }}(q, \dot{q})\right\rangle \\
= & \sqrt{\frac{k_{\mathrm{B}} T \lambda_{1}}{2 \pi \gamma_{11}}} \int d \dot{q}_{1} d q_{\mathrm{b}} d \dot{q}_{\mathrm{b}} \\
& \exp \left[-\frac{1}{2 k_{\mathrm{B}} T}\left\{\frac{\omega^{\ddagger^{2}}}{\lambda_{1} \gamma_{11}} \dot{q}_{1}^{2}+\sum_{j=2}^{n}\left(\dot{q}_{j}^{2}+\omega_{j}^{2} q_{j}^{2}\right)\right\}\right] \\
& {\left[1+\phi_{1}^{\prime}(q, \dot{q}, \gamma, T ; \varepsilon)+\sum_{n=3}^{\infty} \psi_{n}(q, \dot{q}, \gamma, T ; \varepsilon)\right] } \\
& \exp \left[-\frac{1}{2} \phi_{2}(q, \dot{q}, \gamma, T ; \varepsilon)-\frac{U_{\mathrm{NL}}}{k_{\mathrm{B}} T}\right],
\end{aligned}
$$

with the use of the following symbols $\phi_{1}, \phi_{2}, \psi_{n}$ (for $n \geq 3$ ) which are all polynomials of $q$ and $\dot{q}$ by the above procedure:

$$
\begin{aligned}
\frac{m}{\sigma} & =\sqrt{\frac{\lambda_{1}}{k_{\mathrm{B}} T \gamma_{11}}}\left[\dot{q}_{1}+\phi_{1}(q, \dot{q}, \gamma, T ; \varepsilon)\right], \\
\frac{m^{2}}{\sigma^{2}} & =\frac{\lambda_{1}}{k_{\mathrm{B}} T \gamma_{11}} \dot{q}_{1}^{2}+\phi_{2}(q, \dot{q}, \gamma, T ; \varepsilon), \\
\psi_{n}(q, \dot{q}, \gamma, T ; \varepsilon) & =\sqrt{\frac{\gamma_{11}}{k_{\mathrm{B}} T \lambda_{1}}} \frac{1}{n !} \dot{q}_{1} c_{n} H_{n-1}\left(-\frac{m}{\sigma}\right), \\
\phi_{1}^{\prime}(q, \dot{q}, \gamma, T ; \varepsilon) & =\frac{\partial}{\partial \dot{q}_{1}} \phi_{1}(q, \dot{q}, \gamma, T ; \varepsilon) .
\end{aligned}
$$

Note that $\phi_{1}(q, \dot{q}, \gamma, T ; \varepsilon)$ and $\phi_{2}(q, \dot{q}, \gamma, T ; \varepsilon)$ approaches zero as $\varepsilon \rightarrow 0$. In this limit, $\psi_{n}(q, \dot{q}, \gamma, T ; \varepsilon)$ also approaches to zero because the zeroth order part of $y_{1}$ has normal distribution.

We can expand the second exponential in Eq. (37) and obtain a polynomial

$$
\begin{aligned}
& {\left[1+\phi_{1}^{\prime}(q, \dot{q}, \gamma, T ; \varepsilon)+\sum_{n=3}^{\infty} \psi_{n}(q, \dot{q}, \gamma, T ; \varepsilon)\right] } \\
& \exp \left[-\frac{1}{2} \phi_{2}(q, \dot{q}, \gamma, T ; \varepsilon)-\frac{U_{\mathrm{NL}}}{k_{\mathrm{B}} T}\right], \\
= & 1+\sum_{m} B_{m}(\gamma, T ; \varepsilon) q_{2}{ }^{m_{2}} \cdots q_{n}{ }^{m_{n}} \dot{q}_{1}^{m_{n+1}} \cdots \dot{q}_{n}^{m_{2 n}},
\end{aligned}
$$

with the coefficients $B_{m}(\gamma, T ; \varepsilon) \quad$ satisfying $\lim _{\mathcal{E} \rightarrow 0} B_{m}(\gamma, T ; \varepsilon)=0$.

Then, using the following formulas

$$
\begin{aligned}
\int_{-\infty}^{+\infty} \exp \left(-a z^{2}\right) z^{2 \ell} d z & =\sqrt{2 \pi} \frac{(2 \ell-1) ! !}{(2 a)^{(\ell+1 / 2)}} \\
\int_{-\infty}^{+\infty} \exp \left(-a z^{2}\right) z^{2 \ell-1} d z & =0
\end{aligned}
$$

where

$$
(2 \ell-1) ! ! \stackrel{\text { def }}{=}(2 \ell-1)(2 \ell-3) \cdots 3 \cdot 1,
$$

for positive integer $\ell$, and

$$
(-1) ! ! \stackrel{\text { def }}{=} 1,
$$

we obtain

$$
\begin{aligned}
& \left\langle\dot{q}_{1} P_{\text {reaction }}(q, \dot{q})\right\rangle \\
= & \frac{k_{\mathrm{B}} T \lambda_{1}}{\omega^{\ddagger}}\left[\prod_{j=2}^{n}\left(\frac{2 \pi k_{\mathrm{B}} T}{\omega_{j}}\right)\right] \\
& {\left[1+\sum_{m}^{\prime} B_{m}(\gamma, T ; \varepsilon)\left(m_{n+1}-1\right) ! !\left(\frac{\sqrt{k_{\mathrm{B}} T \lambda_{1} \gamma_{11}}}{\omega^{\ddagger}}\right)^{m_{n+1}}\right.} \\
& \left.\prod_{j=2}^{n}\left(m_{n+j}-1\right) ! !\left(\sqrt{k_{\mathrm{B}} T}\right)^{m_{n+j}}\left(m_{j}-1\right) ! !\left(\frac{\sqrt{k_{\mathrm{B}} T}}{\omega_{j}}\right)^{m_{j}}\right],
\end{aligned}
$$

where the sum $\sum_{m}^{\prime}$ is taken over even values of $m$ only. Note that the reactive flux is affected not only by the friction along the reactive coordinate $\left(\gamma_{11}\right)$ but also by that along the nonreactive coordinate $\left(\gamma_{j j}\right.$ with $\left.j=2, \ldots, n\right)$ through the nonlinear couplings.

The denominator in Eq. (27) is obtained in a similar fashion by

$$
\begin{aligned}
& \left\langle\dot{q}_{1}(0) \theta\left(\dot{q}_{1}(0)\right)\right\rangle \\
= & \int d \dot{q}_{1} d q_{\mathrm{b}} d \dot{q}_{\mathrm{b}} \\
& \theta\left(\dot{q}_{1}\right) \dot{q}_{1} \exp \left[-\frac{1}{k_{\mathrm{B}} T}\left\{\frac{1}{2} \dot{q}_{1}^{2}+\sum_{j=2}^{n} \frac{1}{2}\left(\dot{q}_{j}^{2}+\omega_{j}^{2} q_{j}^{2}\right)+U_{\mathrm{NL}}(q)\right\}\right] \\
= & k_{\mathrm{B}} T\left(2 \pi k_{\mathrm{B}} T\right)^{(n-1) / 2} \\
& \int d q_{\mathrm{b}} \exp \left[-\sum_{j=2}^{n} \frac{\omega_{j}^{2}}{2 k_{\mathrm{B}} T} q_{j}^{2}\right] \exp \left[-\frac{U_{\mathrm{NL}}(q)}{k_{\mathrm{B}} T}\right] \\
= & k_{\mathrm{B}} T\left[\prod_{j=2}^{n}\left(\frac{2 \pi k_{\mathrm{B}} T}{\omega_{j}}\right)\right] \\
& {\left[1+\sum_{m}^{\prime} A_{m}(T ; \varepsilon) \prod_{j=2}^{n}\left(m_{j}-1\right) ! !\left(\frac{\sqrt{k_{\mathrm{B}} T}}{\omega_{j}}\right)^{m_{j}}\right] }
\end{aligned}
$$

where

$$
\exp \left[-\frac{U_{\mathrm{NL}}(q)}{k_{\mathrm{B}} T}\right]=\sum_{m} A_{m}(T ; \varepsilon) q_{2}{ }^{m_{2}} \cdots q_{n}{ }^{m_{n}}
$$

is an expansion of $\exp \left[-U_{\mathrm{NL}}(q) / k_{\mathrm{B}} T\right]$ with $q_{1}=0$. In Eq. (44), again, the summation with respect to $m$ is taken over even powers only. Here the TST flux does not depend on any friction exerted by environment.

From Eqs. (27), (43) and (44), and for harmonic approximation $\varepsilon \rightarrow 0$, we have

$$
\lim _{\varepsilon \rightarrow 0} \kappa=\frac{\lambda_{1}}{\omega^{\ddagger}} .
$$

For high viscosity limit, we obtain from Eq. (11)

$$
\kappa \rightarrow \frac{\omega^{\ddagger}}{\gamma_{11}},
$$

which is inversely proportional to the friction coefficient. Thus we can recover the Kramers' high viscosity limit as a special case of harmonic approximation in our nonlinear formulation. 
III. MODEL

As an illustrative example, we employ a model potential by Müller and Brown, ${ }^{70}$ which has three minima and two saddle points. We check the validity and the potential of the new framework in the preceding sections by comparing it with the numerical simulation of the model with several strength of the friction constant. The locations, energies and normal mode frequencies (without friction) of the stationary points are summarized in Table I. For the friction coefficient we set uniform friction $\gamma_{i j}=\gamma \delta_{i j}$.

Numerical values of the transmission coefficient Eq. (27) are obtained by evaluating the integral in Eq. (28) with MonteCarlo sampling ${ }^{71}$ of both the initial condition and the random force. For each initial condition, the trajectory is propagated by the method of Ermak and Buckholz. ${ }^{72}$ The time step of propagation is $10^{-3}$. The system is regarded as captured in the well region when the total energy (the kinetic energy plus the potential) of the system becomes less than $U_{\min }+2 k_{\mathrm{B}} T$, where $U_{\min }$ is the potential at the bottom of each well. The factor 2 is inserted because this system is of two degrees-offreedom.

In this paper we mainly deal with a saddle with higher energy (called "saddle 2" in preceding papers), which connects Minima 1 and 3 and has a significant asymmetrical feature of potential compared to the other saddle, connecting Minima 2 and 3, called "saddle 1." The barrier height of saddle 2 from each minimum is 41 and 107, respectively, which that of saddle 1 is 9 and 36 (in the unit of energy), respectively. ${ }^{64,65}$ In order that the system can be regarded as "captured" at each minimum before visiting the next minimum with the above criterion, the temperature should be less than $\approx 9 / 2$.

\section{RESULTS AND DISCUSSION}

\section{A. Reaction probabilities}

Figure 1 shows the reaction probability as a function of the initial condition of the system $(q, \dot{q})$ in the region of saddle. $q$ and $\dot{q}$ are the normal mode coordinates in the position-space and their velocities, respectively. Here, the initial condition is taken by changing the value of the normal mode reaction coordinate $q_{1}$ while fixing the other three variables $\left(q_{2}, \dot{q}_{1}, \dot{q}_{2}\right)$. In Fig. 1(a), the initial condition is taken as $\left.\left(q_{2}, \dot{q}_{1}, \dot{q}_{2}\right)\right|_{t=0}=$

TABLE I: Name, location, energy and frequencies of the stationary points.

\begin{tabular}{c|ccccc} 
& \multicolumn{5}{|c}{ Normal mode frequencies } \\
Name & $Q_{1}$ & $Q_{2}$ & Energy & Reactive & Nonreactive \\
\hline Minimum1 & -0.558 & 1.442 & -147 & & \\
Minimum2 & 0.623 & 0.028 & -108 & & \\
Minimum3 & -0.050 & 0.467 & -81 & & \\
Saddle1 & 0.212 & 0.293 & -72 & 27.1 & 22.6 \\
Saddle2 & -0.822 & 0.624 & -40 & 27.4 & 22.1
\end{tabular}

(a) Saddle 2, $\left.\left(q_{2}, \dot{q}_{1}, \dot{q}_{2}\right)\right|_{t=0}=(0,0,0)$

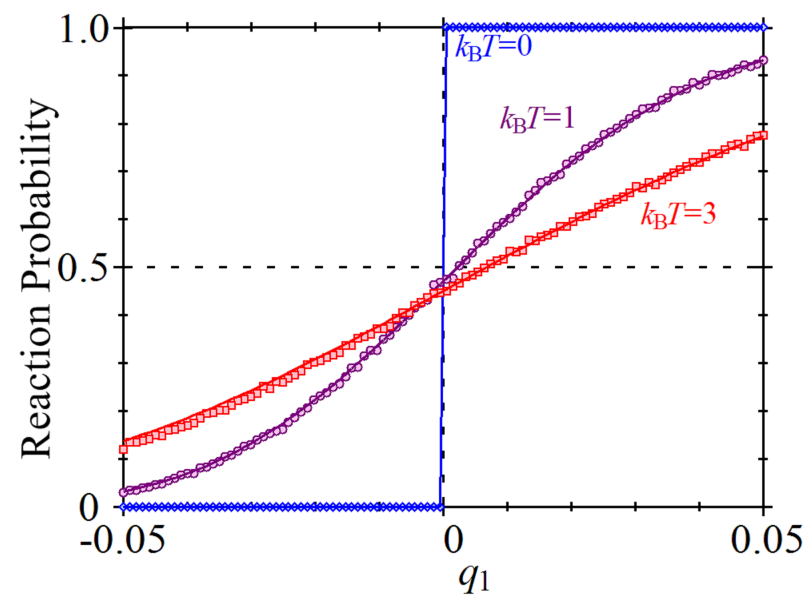

(b) Saddle 1, $\left.\left(q_{2}, \dot{q}_{1}, \dot{q}_{2}\right)\right|_{t=0}=(0.1,0,0)$

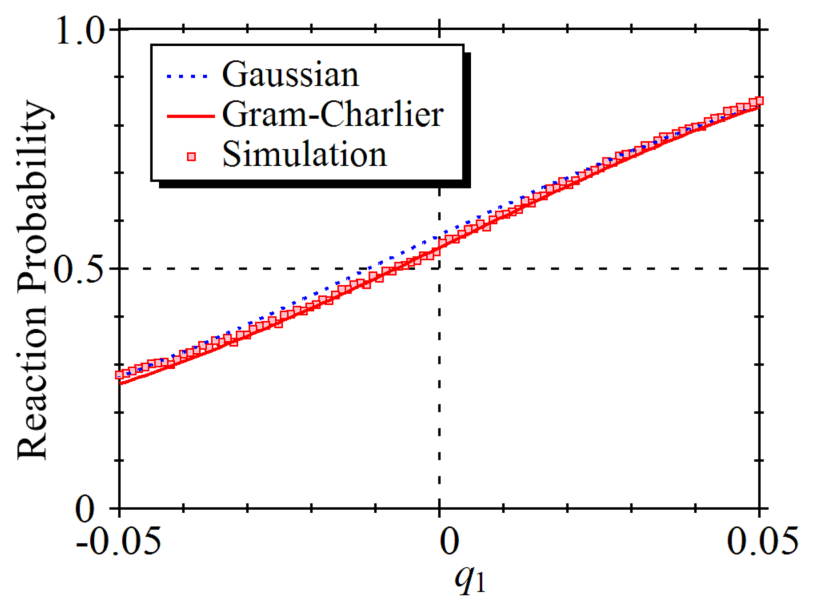

FIG. 1: Reaction probabilities as functions of $q_{1}$ with the initial values of other coordinates indicated. (a) Calculated for saddle 2 of the Müller-Brown potential. Blue diamond: $k_{\mathrm{B}} T=0$, Purple circle: $k_{\mathrm{B}} T=1$, Red square $k_{\mathrm{B}} T=3$. Solid lines show the results of the analytical calculations by Eq. (25) The normal form calculation was performed up to the second order of perturbation. The GramCharlier A series was taken up to the cubic term. (b) Calculated for saddle 1 and $k_{\mathrm{B}} T=3$. Red square: simulation. Blue dotted line: probability distribution of $y_{1}$ is approximated by Gaussian. Red solid line: Gram-Charlier A series was taken up to the cubic term.

$(0,0,0)$ at $k_{\mathrm{B}} T=0,1$, and 3 . The halfway point $P_{\text {reaction }}=1 / 2$ can be regarded as a "boundary of reaction" that divides the space into a "mainly-reactive" $\left(P_{\text {reaction }}>1 / 2\right)$ region and a "mainly-nonreactive" $\left(P_{\text {reaction }}<1 / 2\right)$ one. For $k_{\mathrm{B}} T=0$, the random force is zero [Eq. (2)] and therefore the time evolution of the system is determined uniquely, that is, the reaction probability is either 1 or 0 . The reaction boundary is located at $q_{1}=0$. As the temperature increases, the probability takes intermediate values between 0 and 1 , due to the fluctuation of the random force. The amount of fluctuation 
increases with the temperature. In addition, deviation of the boundary to the positive $q_{1}$ direction is observed for saddle 2 (as we revealed the physical origin of the shift in Ref. 65). The reaction probability based on the new reaction coordinate $y_{1}$ by Eq. (25) proposed in Sec. II B (shown by the solid lines in Fig. 1) reproduces the simulation results satisfactorily including the fluctuation and the migration of the reaction boundary outward to the positive $q_{1}$. Analytical expressions for the second and the third moments used for the calculation are available on-line in supplementary material ${ }^{73}$ to this paper for the case of $\gamma=30$ as an example. For saddle 1 the reaction boundary $P_{\text {reaction }}=1 / 2$ migrates to the negative $q_{1}$ direction as the increase of temperature with the similar initial condition of $\left(q_{2}, \dot{q}_{1}, \dot{q}_{2}\right)$ being zero. ${ }^{65}$ Eq. (25) can also reproduce the results satisfactorily (not shown here).

To illustrate the effect of the deviation from Gaussian distribution of $y_{1}$, we plot the reaction probability for saddle 1 with the initial condition of $\left.\left(q_{2}, \dot{q}_{1}, \dot{q}_{2}\right)\right|_{t=0}=(0.1,0,0)$ at $k_{\mathrm{B}} T=3$. The blue dotted line shows the reaction probability calculated by the Gaussian approximation for $y_{1}$, that is, only the terms in the first bracket in Eq. (25). The red solid line is calculated by the Gram-Charlier A series up to the cubic term $[n=3$ in Eq. (25)]. Note that we can find a small improvement of the approximation by the inclusion of the skewness. For saddle 2, the effect of the skewness was found to be yet smaller, and the calculation with and without the term $n=3$ in Eq. (25) both yielded a good approximation for the reaction probability.

The most striking consequence in these figures is that Eq. 25 is analytical and does not require any trajectory calculation.

\section{B. Transmission coefficients}

In Fig. 2, the transmission coefficients obtained by zeroth (harmonic approximation) and second order perturbation developed in this work, and the numerical simulation are shown as functions of the friction constant $\gamma$. The calculations are performed for Saddle 2 with $k_{\mathrm{B}} T=3,5$. Numerical values are obtained by trajectory calculations with the initial conditions sampled by the Boltzmann distribution on the surface $q_{1}=0$ as in Eq. (28). Hereinafter, we mainly focus on reaction dynamics over saddle 2 because they were found to be more subject to nonlinearity than those over Saddle $1 .^{65}$

As qualitative trends, the transmission coefficients evaluated at several levels of approximation decrease with the friction constant $\gamma$ as the simulation result does. This is due to the increase of the fluctuation of $y_{1}$ as $\gamma$ increases [Eq. (34)], corresponding to increasing number of recrossings with the surface $q_{1}=0$. The deviation of the harmonic approximation from the simulation is already appreciable at $k_{\mathrm{B}} T=3$ and becomes more pronounced at $k_{\mathrm{B}} T=5$ (Fig. 2(a) $\rightarrow$ Fig. 2(b)). This is because the system experiences a wider region on the potential, and hence larger nonlinearity, as the increase of temperature, when passing the saddle. When we incorporate the effect of nonlinearity up to second order in $\varepsilon$ in the framework of minimal NF in Sec. II, the theoretical evaluation by minimal NF better approximates the simulation results than the (a) $k_{\mathrm{B}} T=3$

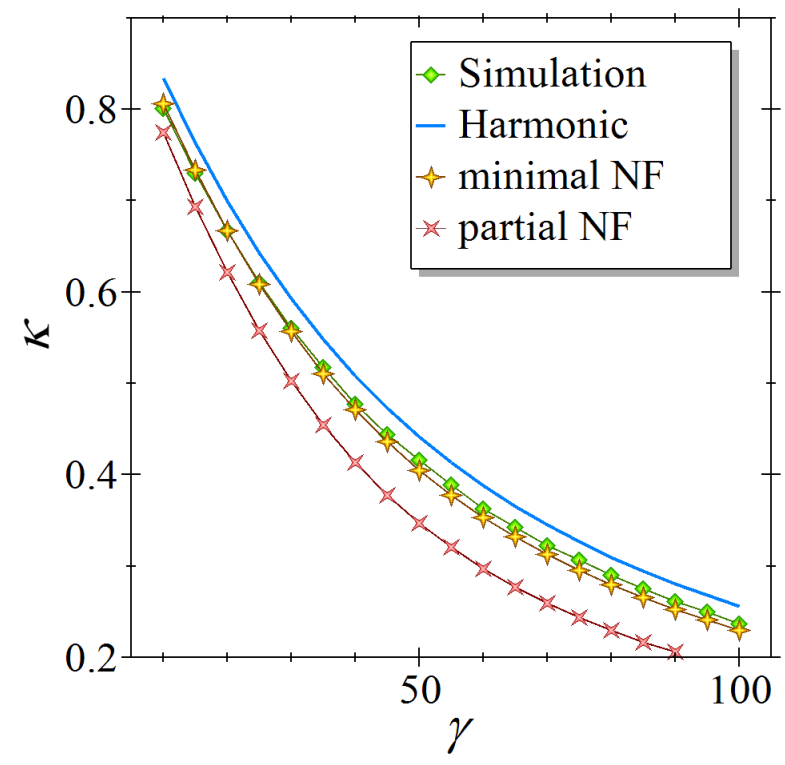

(b) $k_{\mathrm{B}} T=5$

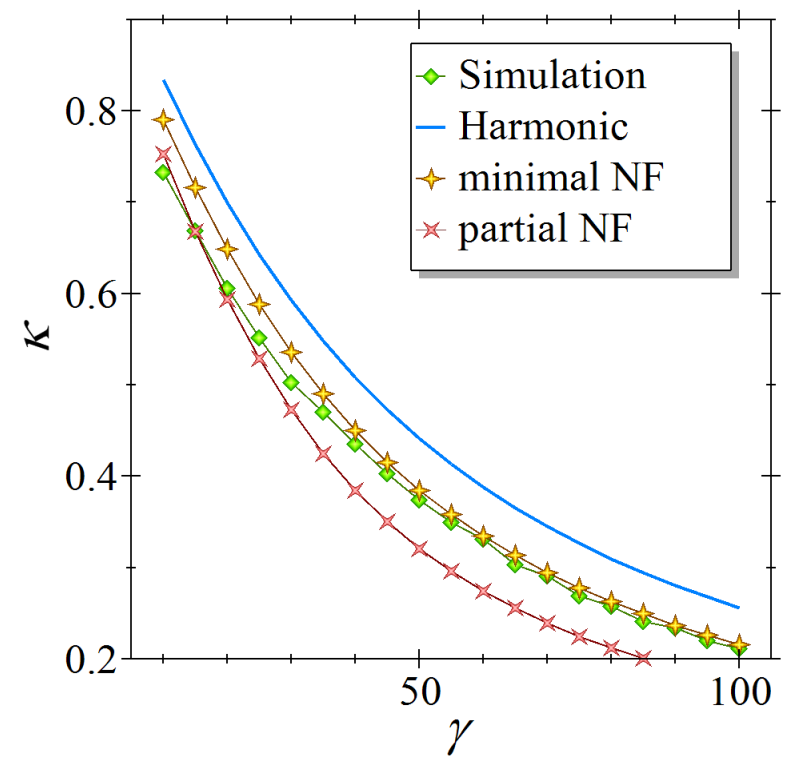

FIG. 2: Transmission coefficients calculated for saddle 2 of the Müller-Brown potential as functions of the friction constant $\gamma$. (a) $k_{\mathrm{B}} T=3$, (b) $k_{\mathrm{B}} T=5$. Green diamond: results of numerical simulations. Blue solid line: calculation by harmonic approximation. Yellow plus: minimal NF up to the second order perturbation. Red cross: partial NF up to the second order perturbation.

harmonic approximation. Note that the partial NF shows poor performance, which is due to the worse convergence property compared with the minimal NF. At the higher temperature $\left(k_{\mathrm{B}} T=5\right)$, the results of minimal NF exhibit some deviations from the simulation, probably due to the insufficient order of the perturbation calculation performed. This shows that the condensed phase reaction probability based on the minimal $\mathrm{NF}$ proposed in this paper is appropriate to evaluate the effect 
(a) $k_{\mathrm{B}} T=3$

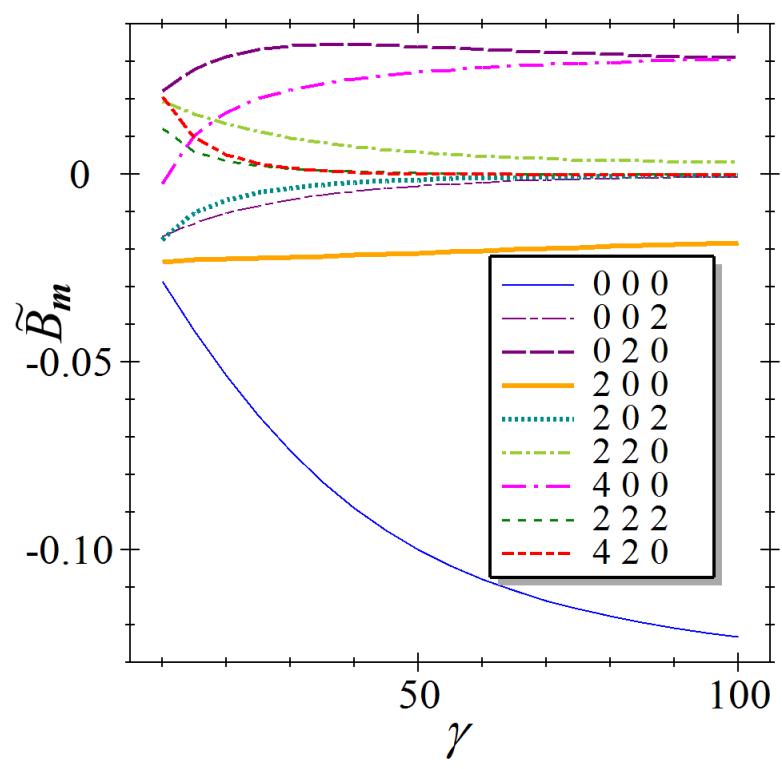

(b) $k_{\mathrm{B}} T=5$

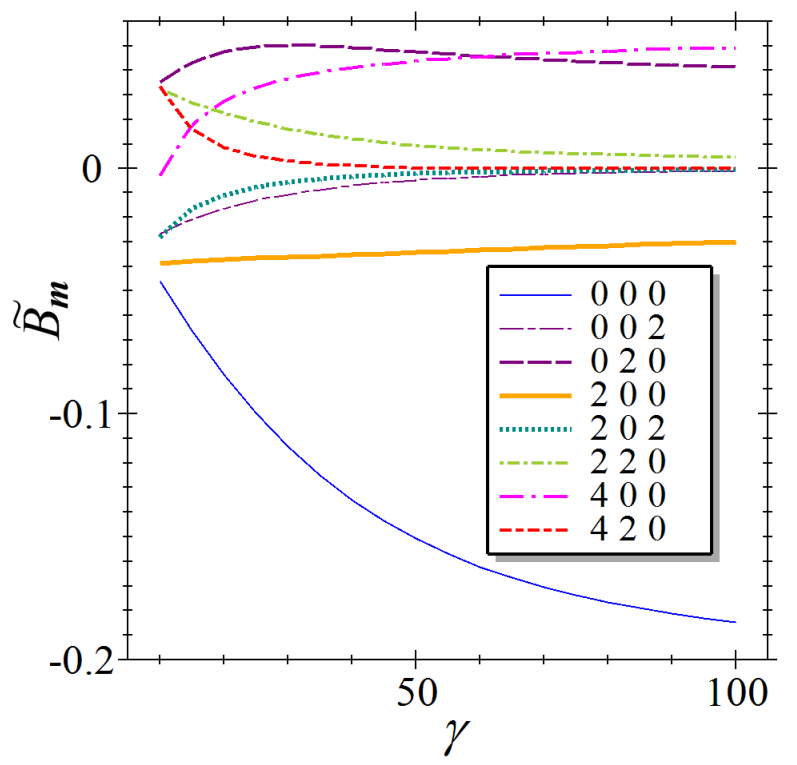

FIG. 3: Values of the coefficients appearing in the polynomial expression of the numerator of the transmission coefficients. (a) $k_{\mathrm{B}} T=$ 3. All the other coefficients not shown are less than 0.012 in magnitude. (b) $k_{\mathrm{B}} T=5$. All the other coefficients not shown are less than 0.02 in magnitude. The inset shows the corresponding powers of $q_{2}, \dot{q}_{1}, \dot{q}_{2}$ for each line.

of nonlinearity on the rate constants of the reactions at such moderately high temperatures. In the followings, our analyses will be all based on the minimal NF approximation.

What is the physical origin to make the actual transmission coefficient $\kappa$ smaller than that of the harmonic approxima- tion? One may anticipate that, as temperature increases, the system is subject to larger nonlinear couplings between the reactive and the nonreactive normal mode coordinates $q$, resulting in recrossings over a surface of $q_{1}=0$ and decreasing $\kappa$, just as in the case of saddle crossing in Hamiltonian systems dependent on the total energy. In condensed phase reactions, there are effects of the environment through the fluctuating force and the friction constants in addition to the nonlinearities, and also their combinations as in Eq. (7). Our theoretical framework presented in this article can identify what the dominant contribution to make the $\kappa$ deviate from the harmonic approximation: We can analyze the contribution of each term appearing in Eqs. (43). Here we define

$$
\begin{aligned}
\tilde{B}_{m} \stackrel{\text { def }}{=} & B_{m}(\gamma, T ; \varepsilon)\left(m_{n+1}-1\right) ! !\left(\frac{\sqrt{k_{\mathrm{B}} T \lambda_{1} \gamma_{11}}}{\omega^{\ddagger}}\right)^{m_{n+1}} \\
& \prod_{j=2}^{n}\left(m_{n+j}-1\right) ! !\left(\sqrt{k_{\mathrm{B}} T}\right)^{m_{n+j}}\left(m_{j}-1\right) ! !\left(\frac{\sqrt{k_{\mathrm{B}} T}}{\omega_{j}}\right)^{m_{j}}(48)
\end{aligned}
$$

which appear in the numerator [Eq. (43)] of the transmission coefficient [Eq. (27)]. Figure 3 shows the value of the coefficient defined above for each combination of the exponents $\left(m_{2}, m_{3}, m_{4}\right)$ of $\left(q_{2}, \dot{q}_{1}, \dot{q}_{2}\right)$, respectively, as functions of the friction constant $\gamma$.

We can see that the main contribution to the decrease of $\kappa$ compared to the harmonic approximation comes from the constant term $B_{0}$ with $\left(m_{2}, m_{3}, m_{4}\right)=(0,0,0)$. Here the word "constant" is used to mean it has no dependence on the variables $\left(q_{2}, \dot{q}_{1}, \dot{q}_{2}\right)$. Note however that it depends on the friction $\gamma$, the temperature $k_{\mathrm{B}} T$, and all the coefficients in the expansion of the potential [Eq. (3)]. For high friction, this term is clearly the dominant. For lower friction, the other terms also make some contribution but the constant term is still significant.

The constant term $B_{0}$ originates only from the constant term $F_{0}$ in the expansion of $y_{1}$ in terms of $(q, \dot{q})$ [Eq. (7)]. To demonstrate the importance of the terms $F_{0}$ in decreasing the $\kappa$ from the harmonic approximation, we perform another calculation on the reaction probability (i.e., $y_{1}>0$ ) by replacing Eq. (7) by

$$
y_{1}=a_{1} q_{1}+a_{2} \dot{q}_{1}-S\left[\lambda_{1}, \tilde{\xi}_{1}\right](t)+F_{0}[\xi](t) .
$$

The resultant transmission coefficients are presented in Fig. 4 at the same temperatures with the same initial conditions. Calculations without $F_{0}$ but with all the other terms in $y_{1}$, that is,

$$
\begin{aligned}
y_{1}= & a_{1} q_{1}+a_{2} \dot{q}_{1}-S\left[\lambda_{1}, \tilde{\xi}_{1}\right](t) \\
& +\sum_{|m| \geq 2} w_{m} q_{1}{ }^{m_{1}} \cdots q_{n}{ }^{m_{n}} \dot{q}_{1}^{m_{n+1}} \cdots \dot{q}_{n}^{m_{2 n}} \\
& +\sum_{|m| \geq 1} F_{m}[\xi](t) q_{1}{ }^{m_{1}} \cdots q_{n}{ }^{m_{n}} \dot{q}_{1}^{m_{n+1}} \cdots \dot{q}_{n}^{m_{2 n}} .
\end{aligned}
$$

in place of Eq. (7) are also plotted in Fig. 4. 
(a) $k_{\mathrm{B}} T=3$

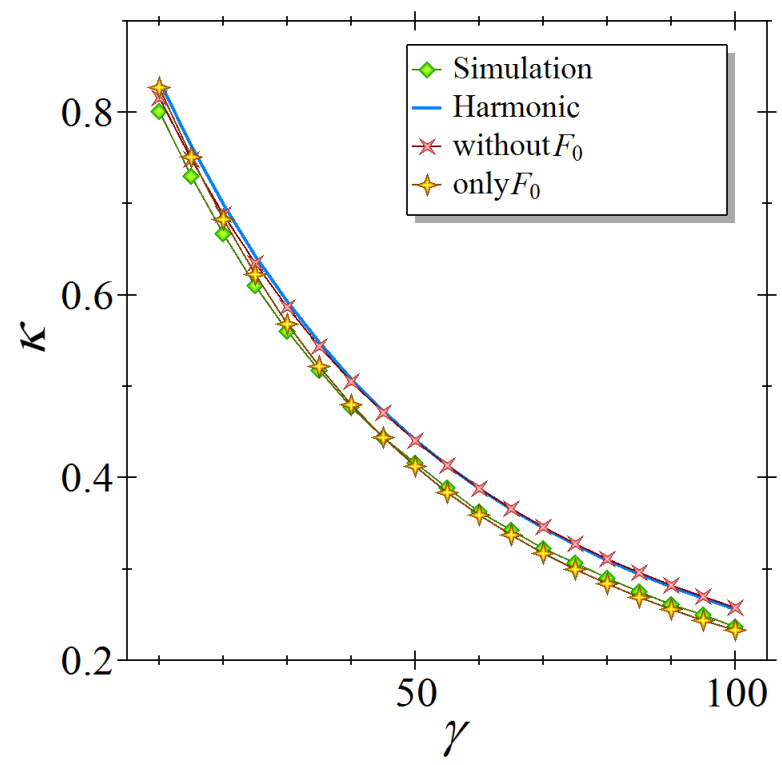

(b) $k_{\mathrm{B}} T=5$

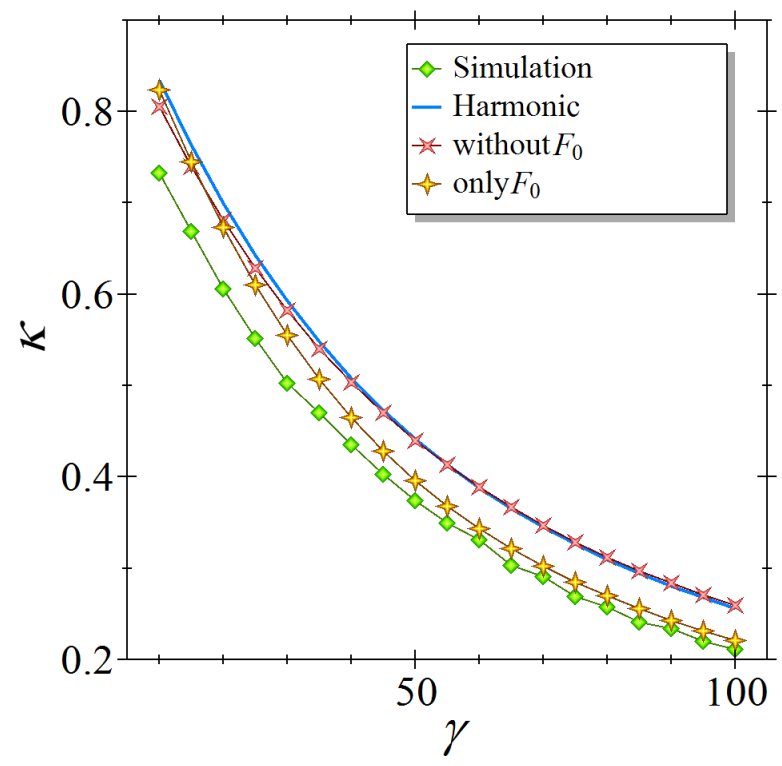

FIG. 4: Transmission coefficients vs friction constant $\gamma$ for saddle 2 of the Müller-Brown potential. (a) $k_{\mathrm{B}} T=3$, (b) $k_{\mathrm{B}} T=5$. Green diamond: results of numerical simulations. Blue solid line: harmonic approximation. Yellow plus: minimal NF adding solely $F_{0}$ to the harmonic approximation in $y_{1}$. Red cross: minimal NF including all the nonlinear contributions excluding $F_{0}$. (a) $k_{\mathrm{B}} T=3$

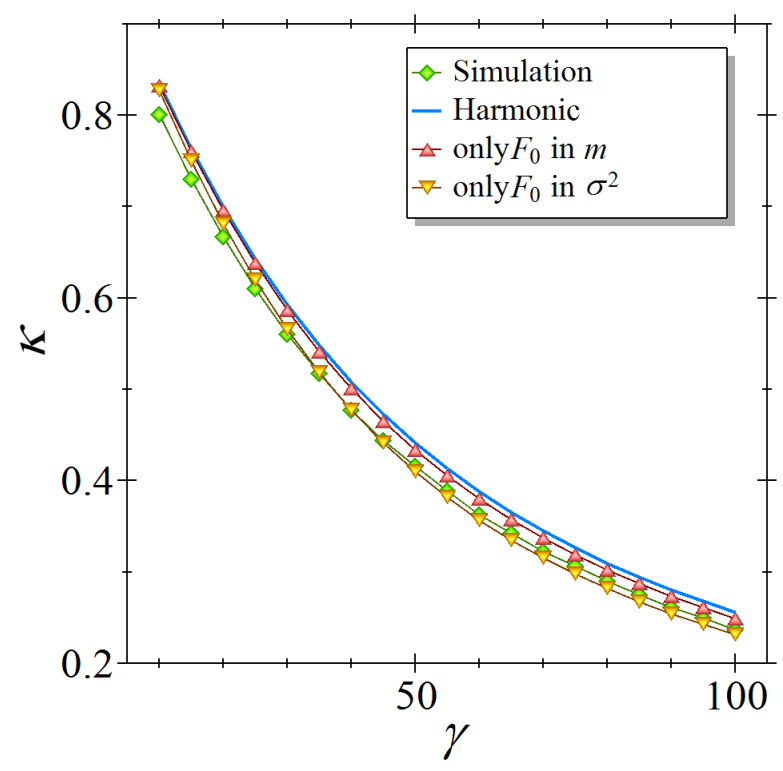

(b) $k_{\mathrm{B}} T=5$

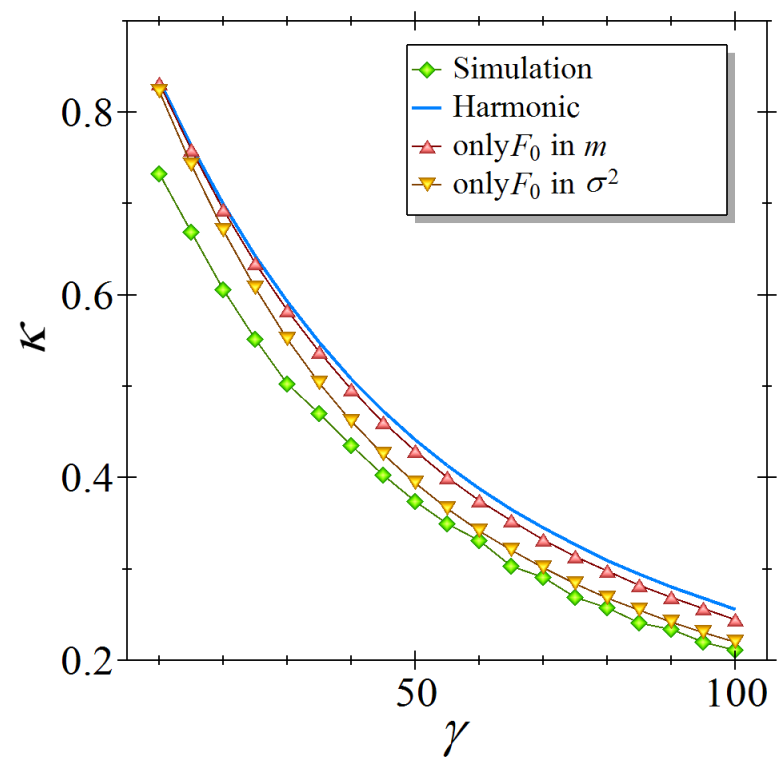

FIG. 5: Transmission coefficients vs friction constant $\gamma$ for saddle 2. (a) $k_{\mathrm{B}} T=3$, (b) $k_{\mathrm{B}} T=5$. Same as Figs. 2 and 4 , but the minimal NF calculations are performed in different ways of the inclusion of the effect of the $F_{0}$. Upper triangle: the minimal NF calculations including the $F_{0}$ only in the mean of the reaction coordinate $y_{1}$. Lower triangle: the minimal NF calculations including the $F_{0}$ only in the variance of the reaction coordinate $y_{1}$. It is interpreted that the increase of the fluctuation is the main cause to make the transmission coefficients small. 
It is seen, especially for large friction, that the calculations including only $F_{0}$ in $y_{1}$ [Eq. (49)] reproduce the simulation results satisfactorily, while those with all the other nonlinear terms excluding $F_{0}$ show almost no improvement from the harmonic approximation. This supports the conclusion that the main contribution to the decrease of $\kappa$ compared to the harmonic approximation comes from the constant term $F_{0}$.

In previous papers, ${ }^{64,65}$ the physical interpretation for $F_{0}$ was given, which arises from both the random force exerted by the environment and nonlinear couplings between the reactive and the nonreactive modes. In the present system of two-degrees of freedom, first the vibration of the nonreactive mode $q_{2}$ is excited by the kick from the environment expressed as the random force along the nonreactive direction $\xi_{2}(t)$. Then such vibrational excitation in the nonreactive mode affects the motion along the reactive mode through nonlinear couplings, making the effective reaction barrier migrate outward to the product direction along the reaction coordinate $q_{1}$ from a surface of $q_{1}=0$. This implies that, even when the system crosses the surface of $q_{1}=0$ from the reactant to the product side, the system more often returns to the reactant region because the effective barrier "escapes" toward the product direction. We can conclude such "combined effect" between the kick by the environment and the nonlinear couplings of the system, yielding the migration of the effective barrier to the product region, is the main contribution to the decrease of the actual transmission coefficient from the harmonic approximation. Although $F_{0}$ also contains the terms nonlinear in $\xi_{1}(t)$, which comes from the nonlinearity along the reactive direction, the contributions from $\xi_{2}(t)$ have been shown to be dominant in the case of saddle $2 .^{65}$

We have shown that the term $F_{0}$ makes the main contribution to decrease the value of $\kappa$ smaller than the harmonic approximation. The next question is then why the transmission coefficient becomes smaller when the term $F_{0}$ exists. The effect originates from the kick by the environment along the nonreactive mode expressed as the random force $\xi_{2}(t)$. Therefore the term $F_{0}$ has its own statistics such as mean and variance in the ensemble of all realizations of the random force. Here we investigate whether the term $F_{0}$ affects the transmission coefficient through the mean or the variance. To this end, we further perform calculations in which the mean of the reaction coordinate $y_{1}$ is evaluated by the expression with $F_{0}$ [Eq. (49)], while the variance $\sigma^{2}$ is approximated by the harmonic part:

$$
y_{1}=a_{1} q_{1}+a_{2} \dot{q}_{1}-S\left[\lambda_{1}, \tilde{\xi}_{1}\right](t),
$$

resulting in only the first term in Eq. (34). We also perform the other way, that is, the mean of the reaction coordinate $y_{1}$ is evaluated by harmonic approximation [Eq. (51)], resulting in only the first term in Eq. (33), while the variance $\sigma^{2}$ is evaluated by the expression with $F_{0}$ [Eq. (49)]. The results are shown in Fig. 5. It is seen that the variance $\sigma^{2}$ carries the larger contribution to the transmission coefficient than the mean $m$. Physical interpretations can be given as follows: In Eq. (49), the term $-S\left[\lambda_{1}, \tilde{\xi}_{1}\right](t)$ expresses the effect of the direct kick along the reactive direction by the random force from the environment. Its fluctuation, which increases with increas- ing $\gamma$, causes recrossings with the dividing surface. This explains the decrease of $\kappa$ with $\gamma$ in the harmonic approximation. The additional term $F_{0}[\xi](t)$ in Eq. (49), coming from the nonlinearities, also has a fluctuation due to the fluctuation of $\xi_{2}(t)$ (recall that the $F_{0}[\xi](t)$ expresses mainly the effect of the random force along the nonreactive mode affecting the reaction through the couplings). Due to the additional fluctuation, recrossings with the dividing surface increase, resulting in the decrease of the reaction probability.

In Fig. 3, it is seen that the contribution from the terms with nonzero powers of velocities $\left(\dot{q}_{1}\right.$ or $\left.\dot{q}_{2}\right)$ decay with increasing friction $\gamma$, except for the term $\dot{q}_{1}^{2}$. [Recall that the contribution $\tilde{B}_{m}$ to the transmission coefficient with the combination $\left(m_{2}, m_{3}, m_{4}\right)$ comes from the coefficient of $q_{2}^{m_{2}} \dot{q}_{1}^{m_{3}} \dot{q}_{2}^{m_{4}}$ in the polynomial expansion of the integrand Eq. (39)]. This fact corresponds to the transition to the overdamped regime where the effect of initial velocity to the motion becomes negligible due to fast damping. On the other hand, the term $\dot{q}_{1}^{2}$ comes from the Taylor expansion of the reaction probability [or, strictly speaking, the derivative of the reaction probability, since we use integration by parts in Eq. (31)]. The reaction probability is expressed in terms of $m / \sigma$ [Eq. (21)], where the main part of $m$ is $\dot{q}_{1}$ [Eq. (33)] and the fluctuation $\sigma$ has a contribution from the nonlinear term $F_{0}$. Thus the polynomial expansion in Eq. (39) contains terms of $\dot{q}_{1}^{2}$ with the coefficients reflecting the effect of the fluctuation of $F_{0}$, which does not decay as $\gamma$ increases as can be seen by the $(0,0,0)$-term in Fig. 3.

\section{SUMMARY AND OUTLOOK}

A framework to calculate the rate constants of the chemical reaction in condensed phase was presented. With the multidimensional underdamped Langevin equation, we treated explicitly the effect of fluctuating environment (through friction and random force) and the nonlinearity in the potential of mean force. The reaction probability is analytically obtained as the probability that the reaction coordinate (buried in thermal fluctuation and nonlinearity of the system) takes positive values. The integration with the Boltzmann distribution of the initial conditions in the region of saddle leads to the reaction rate constant. The physical origin of the rate can be led by the analyticity of the present formulation. The contribution to the reactivity (the sign of the reaction coordinate $y_{1}$ ) can be classified into the linear part, the (direct) environment (e.g., solvent) effect, the nonlinearity intrinsic to the system (e.g., solute), and the combination of environment and nonlinearity. The rate, which is statistical property, can be understood in terms of the statistical properties of each component such as the average, the variance, and the higher moments.

As an example, we illustrated the present scheme by analyzing the model potential of Müller-Brown as the potential of mean force with a range of friction constant. The transmission coefficient $\kappa$ was found to be smaller than the harmonic approximation. The physical origin was identified as arising from the $F_{0}$ component that represents one of the nonlinear cooperation effects between the nonlinearity of the system and 
the random force: The vibration along the nonreactive mode is excited by kick from the surroundings, which then affects the reactive mode through the nonlinear couplings. This effect has its own fluctuation due to the random force. The additional fluctuation causes an increase in the number of recrossings, resulting in a decrease of the rate.

Although the $F_{0}$ component was turned out to be dominant in the present example, the other components in the expression of the reaction coordinate $y_{1}$ can in general contribute to the rate constant, and the amount of each contribution clearly depends on the kinds of system and the conditions (i.e., temperature and friction constant). The present method is expected to provide a useful tool for understanding the physical origin of the reaction rate in fluctuating environment.

In the pioneering work by Kramers ${ }^{58}$ he found that the rate constant becomes proportional to the inverse of the friction constant $\gamma$ in the case of high viscosity whilst it becomes proportional to the $\gamma$ in the case of low viscosity, resulting in a turn over of the rate constant as a function of the $\gamma$. The correspondence of our theory to Kramers' low viscosity limit is not straightforward because the rate determining step in the limit is the excitation in the reactant well (energy or action space diffusion). Furthermore, his original treatment of the low viscosity limit is based on one degree of freedom Hamiltonian, very weakly coupled with the environment, in which the constant of action is well defined through the whole space from the reactant to the product. However, in the case of many degrees of freedom systems, the very existence of the constants of action through the whole phase space is far from trivial due to the existence of chaos (nonlinear resonance) of the system. ${ }^{74-76}$ Thus the assumption of the Boltzmann distribution, corresponding to the sufficiently fast vibrational relaxation in the well region compared to the time scale of passage through the saddle, may need further investigations. For Hamiltonian systems, concepts of resonance overlap ${ }^{77}$ has been developed concerning the vibrational energy transfer in the well region. Also, the investigation of area-preserving Poincaré map ${ }^{9}$ has found a phase space separatrix functioning as a "transition state." Unfortunately no theory is known for the extension of these works to non-Hamiltonian, dissipative systems with an external time-dependent driving force. It would be interesting to seek for such extensions.

Another possible extension of the present theory is to the case of colored noise. If the time scale of the motions determining the reaction dynamics overlaps with the time scale of solvent motion, the autocorrelation function of the fluctuating force is no longer approximated by the delta function but the values of the force at different times are correlated. In such cases, the fluctuation-dissipation theorem demands that the equation of motion should be replaced by a generalized Langevin equation with an appropriate memory kernel. ${ }^{78,79}$ We will report this extension elsewhere in a separate paper.

\section{ACKNOWLEDGMENTS}

This work has been supported by Research Fellowships of the Japan Society for the Promotion of Science for Young Scientists (to SK) and by JSPS, JST/CREST, Priority Area 'Molecular Theory for Real Systems' (to TK).
${ }^{1}$ C. Jaffe, S. D. Ross, M. W. Lo, J. Marsden, D. Farrelly and T. Uzer, Phys. Rev. Lett., 2002, 89, 011101_1-011101_4.

2 F. Gabern, W. S. Koon, J. E. Marsden and S. D. Ross, Physica D, 2005, 211, 391-406.

3 À. Jorba, Experimental Mathematics, 1999, 8, 155-195.

4 T. Uzer, C. Jaffé, J. Palacián, P. Yanguas and S. Wiggins, Nonlinearity, 2002, 15, 957-992.

5 S. Kawai, C. Jaffé and T. Uzer, J. Phys. B: At. Mol. Opt. Phys., 2005, 38, S261.

6 T. Komatsuzaki and R. S. Berry, J. Chem. Phys., 1999, 110, 91609173.

7 M. Pilling and P. Seakins, Reaction Kinetics, Oxford University Press, Oxford, 1995.

8 M. Karplus, J. Phys. Chem. B, 2000, 104, 11-27.

9 M. J. Davis and S. K. Gray, J. Chem. Phys., 1986, 84, 5389.

10 S. Kawai, H. Teramoto, C.-B. Li, T. Komatsuzaki and M. Toda, Adv. Chem. Phys., 2009.

11 T. Komatsuzaki, A. Baba, S. Kawai, M. Toda, J. Straub and R. Berry, Adv. Chem. Phys., 2009.

12 H. Eyring, J. Chem. Phys., 1935, 3, 107-115.

13 E. Wigner, J. Chem. Phys., 1937, 5, 720-725.

14 M. G. Evans and M. Polanyi, Trans. Faraday Soc., 1935, 31, 875894.

15 O. K. Rice and H. C. Ramsperger, J. Am. Chem. Soc., 1928, 50, 617-620.

16 L. S. Kassel, J. Phys. Chem., 1928, 32, 1065-1079.
17 R. A. Marcus, J. Chem. Phys., 1952, 20, 359-364.

18 J. C. Keck, Adv. Chem. Phys., 1967, 13, 85-121.

19 D. G. Truhlar and B. C. Garrett, Acc. Chem. Res., 1980, 13, 440448.

20 T. Komatsuzaki and M. Nagaoka, J. Chem. Phys., 1996, 105, 10838.

21 T. Komatsuzaki and M. Nagaoka, Chem. Phys. Lett., 1997, 265, 91.

22 T. Komatsuzaki and R. S. Berry, Phys. Chem. Chem. Phys., 1999, $1,1387$.

23 T. Komatsuzaki and R. S. Berry, J. Chem. Phys., 2001, 115, 4105.

24 T. Komatsuzaki and R. S. Berry, J. Mol. Struct. (Theochem), 2000, 506, 55.

25 T. Komatsuzaki and R. S. Berry, Proc. Nat. Acad. Sci. USA, 2001, 98, 7666.

26 T. Komatsuzaki and R. S. Berry, J. Phys. Chem. A, 2002, 106, 10945.

27 S. Wiggins, L. Wiesenfeld, C. Jaffé and T. Uzer, Phys. Rev. Lett., 2001, 86, 5478-5481.

28 H. Waalkens, A. Burbanks and S. Wiggins, J. Chem. Phys., 2004, 121, 6207.

29 H. Waalkens, A. Burbanks and S. Wiggins, J. Phys. A, 2004, 37, L257-L265.

30 H. Waalkens and S. Wiggins, J. Phys. A, 2004, 37, L435-L445.

31 S. Kawai, Y. Fujimura, O. Kajimoto, T. Yamashita, C.-B. Li, T. Komatsuzaki and M. Toda, Phys. Rev. A, 2007, 75, 022714. 
32 C.-B. Li, Y. Matsunaga, M. Toda and T. Komatsuzaki, J. Chem. Phys., 2005, 123, 184301.

33 C.-B. Li, A. Shojiguchi, M. Toda and T. Komatsuzaki, Few-Body Systems, 2006, 38, 173.

34 C.-B. Li, A. Shojiguchi, M. Toda and T. Komatsuzaki, Phys. Rev. Lett., 2006, 97, 028302.

35 C.-B. Li, M. Toda and T. Komatsuzaki, J. Chem. Phys., 2009, 130, 124116.

36 T. Komatsuzaki and R. S. Berry, Adv. Chem. Phys., 2002, 123, 79.

37 M. Toda, Adv. Chem. Phys., 2002, 123, 153.

38 T. Komatsuzaki and R. S. Berry, Adv. Chem. Phys., 2005, 130, 143.

39 C. Jaffé, S. Kawai, J. Palacián, P. Yanguas and T. Uzer, $A d v$. Chem. Phys., 2005, 130, 171.

40 M. Toda, Adv. Chem. Phys., 2005, 130, 337.

41 T. Bartsch, J. M. Moix, R. Hernandez, S. Kawai and T. Uzer, $A d v$. Chem. Phys., 2008, 140, 191-238.

42 H. Waalkens, R. Schubert and S. Wiggins, Nonlinearity, 2008, 21, R1-R118.

43 Geometrical Structures of Phase Space in Multidimensional Chaos: Applications to Chemical Reaction Dynamics in Complex Systems, Adv. Chem. Phys., ed. M. Toda, T. Komatsuzaki, T. Konishi, R. S. Berry and S. A. Rice, Wiley, 2005, vol. 130A,130B.

44 R. S. Berry, T. Komatsuzaki and D. M. Leitner eds. Kinetics and Nonlinear Dynamics of Complex ManyBody Systems, Adv. Chem. Phys. in press.

45 A. Lichtenberg and M. Lieberman, Regular and Chaotic Dynamics, Springer, 1992.

46 E. R. Lovejoy, S. K. Kim and C. B. Moore, Science, 1992, 256, 1541-1544.

47 E. R. Lovejoy and C. B. Moore, J. Chem. Phys., 1993, 98, 78467854.

48 W. H. Miller, Faraday Discussions Chem. Soc., 1977, 62, 40-46.

49 T. Seideman and W. H. Miller, J. Chem. Phys., 1991, 95, 1768.

50 S. Keshavamurthy and W. H. Miller, Chem. Phys. Lett., 1993, 205, 96.

51 R. Hernandez and W. H. Miller, Chem. Phys. Lett., 1993, 214, 129-136.

52 R. Hernandez, J. Chem. Phys., 1994, 101, 9534.

53 R. A. Marcus, Science, 1992, 256, 1523-1524.

54 D. J. Wales and R. S. Berry, J. Phys. B, 1991, 24, L351.

55 R. J. Hinde, R. S. Berry and D. J. Wales, J. Chem. Phys., 1992, 96, 1376
56 C. Amitrano and R. S. Berry, Phys. Rev. Lett., 1992, 68, 729-732.

57 R. J. Hinde and R. S. Berry, J. Chem. Phys., 1993, 99, 2942-2963.

58 H. A. Kramers, Physica, 1940, 7, 284-304.

59 R. F. Grote and J. T. Hynes, J. Chem. Phys., 1980, 73, 2715-2732.

60 H. Sumi and R. A. Marcus, J. Chem. Phys., 1986, 84, 4894-4914.

61 H. Sumi, J. Phys. Chem., 1991, 95, 3334-3350.

62 A. Y. T. Leung and Q. C. Zhang, J. Sound Vib., 2003, 266, 261279.

63 S. Kawai, A. D. Bandrauk, C. Jaffé, T. Bartsch, J. Palacián and T. Uzer, J. Chem. Phys., 2007, 126, 164306_1-164306_12.

64 S. Kawai and T. Komatsuzaki, J. Chem. Phys., 2009, 131, 224505.

65 S. Kawai and T. Komatsuzaki, J. Chem. Phys., 2009, 131, 224506.

66 S. Kawai and T. Komatsuzaki, Phys. Chem. Chem. Phys., submitted for publication.

67 H. Cramér, Mathematical Methods of Statistics, Princeton University Press, Princeton, 1946.

68 J. T. Hynes, The Theory of Chemical Reaction Dynamics, CRC, Boca Raton, FL, 1985, vol. IV, pp. 171-234.

69 B. J. Gertner, K. R. Wilson and J. T. Hynes, J. Chem. Phys., 1989, 90, 3537-3558.

70 K. Müller and L. D. Brown, Theor. Chim. Acta, 1979, 53, 75-93.

71 W. H. Press, S. A. Teukolsky, W. T. Vetterling and B. P. Flannery, Numerical Recipes in C, Cambridge University Press, Cambridge, 1992.

72 D. L. Ermak and H. Buckholz, J. Comp. Phys., 1980, 35, 169182.

73 See AIP Document No. E-PAPS: XXXX for the full explicit expressions of the results of NF calculations. E-PAPS document files may be retrieved free of charge from AIP's FTP server http://www.aip.org/pubservs/paps.html or from ftp.aip.org in the directory /epaps/. For further information: e-mail: paps@aip.org or fax: 516-576-2223.

74 R. B. Shirts and W. P. Reinhardt, J. Chem. Phys., 1982, 77, 15.

75 H. Teramoto and T. Komatsuzaki, Phys. Rev. E, 2008, 78, 017202.

76 H. Teramoto and T. Komatsuzaki, J. Chem. Phys., 2008, 129, 094302.

77 D. W. Oxtoby and S. A. Rice, J. Chem. Phys., 1976, 65, 1676.

78 T. Bartsch, T. Uzer and R. Hernandez, J. Chem. Phys., 2005, 123, 204102 1-204102 14.

79 R. Zwanzig, Nonequilibrium Statistical Mechanics, Oxford University Press, London, 2001. 